\title{
Outcomes of ring versus suture annuloplasty for tricuspid valve repair in patients undergoing mitral valve surgery
}

\author{
Sung Ho Shinn, MD, PhD, ${ }^{a}$ Victor Dayan, MD, PhD, ${ }^{a}$ Hartzell V. Schaff, MD, ${ }^{b}$ Joseph A. Dearani, MD, ${ }^{b}$ \\ Lyle D. Joyce, MD, PhD, ${ }^{b}$ Brian Lahr, MS, ${ }^{b}$ Kevin L. Greason, MD, ${ }^{a}$ John M. Stulak, MD, ${ }^{a}$ and \\ Richard C. Daly, MD
}

\begin{abstract}
Objective: There is controversy regarding the comparative effectiveness of methods of tricuspid valve (TV) repair-prosthetic ring versus suture annuloplasty_in patients undergoing operation for primary mitral valve (MV) disease. In this study, we analyzed factors associated with patient survival and recurrent tricuspid regurgitation (TR) following TV repair and focused on results stratified by method of tricuspid valve repair.
\end{abstract}

Methods: We reviewed patients who underwent TV repair with suture (De Vega) or flexible ring annuloplasties at the time of MV surgery from 1995 to 2010. Patients with prior cardiac or concomitant aortic valve operations were excluded. Propensity matching was performed to account for potential differences in baseline characteristics between the groups. Primary outcomes were long-term mortality and postoperative TR grade.

Results: In the overall study, there were 415 patients with median age 72 years (range, 63-78 years), from which 148 matched pairs were identified by propensity score analysis. In the overall cohort, patients in the ring annuloplasty group more often had preoperative transvenous pacemakers $(P=.05)$, lower ejection fractions $(P=.028)$, and more recent years of operation $(P<.001)$. For patients who had De Vega suture annuloplasty, long-term mortality was not different from that of patients who had ring annuloplasty (hazard ratio, $0.93 ; 95 \%$ confidence interval, $0.67-1.30$ ). Older age, preoperative diabetes, and preoperative right ventricular dysfunction were predictors for long-term mortality. Durability of the annuloplasty methods was similar with no significant difference in trend of recurrent TR grades over follow-up $(P=.807)$. Etiology of mitral regurgitation was not associated with recurrent TR during follow-up $(P=.857)$.

Conclusions: Late survival and TV durability following concomitant TV repair during MV surgery did not differ with respect to TV repair technique. In this series of patients with repaired tricuspid valves, etiology of MV disease did not influence postoperative changes in TR. (J Thorac Cardiovasc Surg 2016;152:406-15)

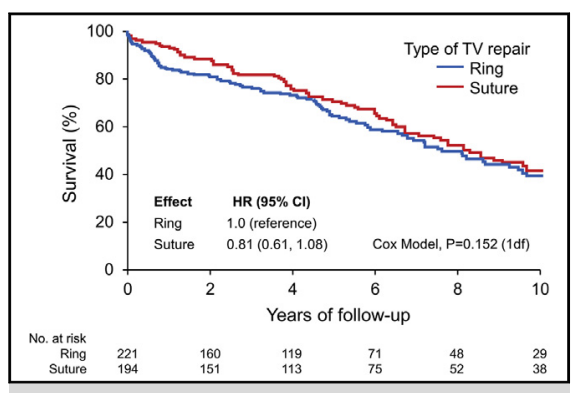

Probability of being in different tricuspid regurgitation grades after ring or De Vega suture annuloplasty.

\section{Central Message}

Outcomes of concomitant tricuspid valve repair during mitral valve surgery are similar with De Vega suture annuloplasty and flexible ring annuloplasty.

\section{Perspective}

The effectiveness of concomitant tricuspid valve repair using ring versus De Vega suture annuloplasty is debated. In the present study of patients undergoing mitral valve surgery and concomitant tricuspid valve repair, we found similar outcomes regarding patient survival and repair durability irrespective of tricuspid valve repair technique. Etiology of mitral valve disease did not influence residual or recurrent tricuspid regurgitation.

See Editorial Commentary page 416.
Functional tricuspid regurgitation (TR) is relatively common in association with left-sided heart disease, especially mitral valve (MV) disease. In most instances, tricuspid

From the ${ }^{\mathrm{a} D e p a r t m e n t}$ of Cardiovascular Surgery and ${ }^{\mathrm{b}}$ Division of Biomedical Statistics and Informatics, Mayo Clinic, Rochester, Minn.

S.H. Shinn is currently with Department of Thoracic and Cardiovascular Surgery, Seoul Eulji Hospital, Eulji University, Seoul, South Korea.

V.D. is currently with Department of Cardiac Surgery, Centro Cardiovascular Universitario, Montevideo, Uruguay.

Received for publication Aug 26, 2015; revisions received April 5, 2016; accepted for publication April 14, 2016; available ahead of print June 1, 2016.

Address for reprints: Hartzell V. Schaff, MD, Department of Cardiovascular Surgery, Mayo Clinic, 200 First Street SW, Rochester, MN 55905 (E-mail: schaff@ mayo.edu). $0022-5223 / \$ 36.00$

Copyright $(2) 2016$ by The American Association for Thoracic Surgery

http://dx.doi.org/10.1016/j.jtcvs.2016.04.068 valve (TV) leaflets are morphologically normal but do not coapt properly because of distortion of leaflets, chords, and papillary muscles resulting from dilation of the right ventricle (RV) and TV annulus. The best method of correcting functional TR is uncertain. McCarthy and colleagues ${ }^{1}$ reported that ring annuloplasty was superior to suture annuloplasty in patients with functional TR, but there are many

Scanning this QR code will take you to supplemental figure and tables for this article. 


$$
\begin{aligned}
& \text { Abbreviations and Acronyms } \\
& \begin{aligned}
\text { COPD } & =\text { chronic obstructive pulmonary disease } \\
\text { MR } & =\text { mitral regurgitation } \\
\text { MV } & =\text { mitral valve } \\
\text { NYHA } & =\text { New York Heart Association } \\
\text { RV } & =\text { right ventricle } \\
\text { RVSP } & =\text { right ventricle systolic pressure } \\
\text { TR } & =\text { tricuspid regurgitation } \\
\text { TTE } & =\text { transthoracic echocardiography } \\
\text { TV } & =\text { tricuspid valve }
\end{aligned}
\end{aligned}
$$

confounding factors, including variable etiologies of left heart disease and lack of standardization of surgical techniques. $^{2,3}$ These potentially confounding factors are also present in systematic reviews and meta-analyses. ${ }^{4,5}$ Therefore, we sought to reduce these potential biases by focusing on surgical management of functional TR in patients undergoing MV operations.

Important aims of the investigation were evaluation of outcome according to surgical method (suture vs ring annuloplasty) and exploration of the effect of pathology of MV disease on late survival and recurrence of TR.

\section{METHODS}

\section{Study Population}

The investigation was approved by the institutional review board of the Mayo Clinic, and all patients gave research authorization. The institutional database was searched for patients who underwent MV replacement or repair with concomitant TV repair from January 1995 to December 2010. During the study period, TV repair was performed in patients with severe (grade 4) TR as determined by preoperative Doppler echocardiography and in patients with preoperative signs of right heart failure and lesser degrees of TR by echocardiography. To minimize confounding bias, we excluded the patients with previous cardiac operations and those with concomitant aortic valve surgery. We also excluded patients with endocarditis, organic TV disease, and those with congenital anomalies. Only patients in whom tricuspid repair was performed with suture (De Vega) ${ }^{6,7}$ or flexible ring annuloplasty were included. Rigid rings have not been used for TV repair at our clinic since 2004. Chronic obstructive pulmonary disease (COPD) was defined according to Society of Thoracic Surgeons criteria, which are based on spirometry results (forced expiratory volume in 1 second $<75 \%$ predicted) and/or requirement for bronchodilator therapy or steroid therapy.

\section{Surgical Procedure}

Most operations were performed through a median sternotomy with standard cardiopulmonary bypass at normothermia or mild hypothermia. Myocardial protection was achieved with antegrade and/or retrograde cold blood cardioplegia.

\section{Follow-up}

Vital status was determined through the Mayo Clinic registration database, which collates information from multiple sources, including correspondence from family and physicians and ACCURINT, an institutionally approved web-based resource and location service (www.accurint.com). ACCURINT is a subsidiary of Seisint Inc (Boca Raton, Fla), a private information management company that provides access to social security death data and a number of other data sources. Such sources have been commonly used to provide death information. ${ }^{8}$ Echocardiographic reports were reviewed, and New York Heart Association (NYHA) functional class was determined from clinical records and responses to surveys sent to all patients 1, 3, 5 , and 10 years postoperatively. Postoperative morbidities were analyzed according to the guidelines for reporting morbidity and mortality after cardiac valve operations approved by the Society of Thoracic Surgeons. ${ }^{9}$

\section{Assessment of Follow-up TR Change}

The trend of TR grades after TR repair was evaluated by transthoracic echocardiography (TTE). TTE was performed routinely before discharge and at the discretion of referring physicians during follow-up. Only follow-up (not predischarge) TTE results were included in the analyses. Interpretations of postoperative echocardiograms were obtained at as many time points as available for each patient.

Comprehensive echocardiographic evaluations completed at our clinic were performed according to the American Society of Echocardiography guidelines. ${ }^{10}$ Briefly, TR was graded 0 for no regurgitation, $1+$ for mild, $2+$ for moderate, $3+$ for moderate to severe, and $4+$ for severe. Positive signs of severe TR included vena contracta of TR jet $>7 \mathrm{~mm}$, persistent dilation of inferior vena cava during the respiratory cycle, and systolic reversal flow in the hepatic veins. Right ventricular size was qualitatively graded in the apical 4-chamber and subcostal views considering its relative size to the left ventricle chamber. Right ventricular systolic function was assessed by experienced echocardiographers as normal or reduced considering visually estimated RV global contractility and regional wall motion. Peak RV systolic pressure (RVSP) was estimated by continuous wave Doppler of the TR jet using the modified Bernoulli equation and right atrial pressure, which was estimated according to inferior vena cava size and presence of plethora The grade of mitral regurgitation (MR) was evaluated according to the MR effective orifice area, regurgitant volume, and the other supportive findings. ${ }^{11}$

\section{End Points and Analysis}

In the data analysis we addressed 3 questions. First, does the method of annuloplasty repair (suture vs ring) affect patient outcome and durability of repair? Second, is there any difference of outcome of TV repair based on the etiology of MV disease (ie, rheumatic, ischemic, and degenerative). And third, what are the risk factors for overall mortality and postoperative residual or recurrent TR following TV repair?

\section{Statistical Methods}

Descriptive statistics on baseline variables were presented as frequency (percentage) for categorical variables and as quartiles (median, 25th, and 75th percentiles) for continuous variables. Baseline differences between suture and ring treatment groups were determined by $\chi^{2}$ test, Fisher exact test, or Wilcoxon rank sum test, as appropriate. Survival curves were constructed using the Kaplan-Meier product limit estimator, and median follow-up time was estimated using the reverse Kaplan-Meier method in which the event indicator in the standard calculation is reversed as if censoring were the outcome. The association of baseline variables (eg, age, body surface area, hypertension, diabetes, COPD, renal failure, preoperative ejection fraction, preoperative right ventricular dysfunction size, and MV etiology) with survival time was analyzed with Cox proportional hazards $(\mathrm{PH})$ regression modeling, from which hazard ratios (HRs) and $95 \%$ confidence intervals (CIs) are reported to describe effects. The model assumption of $\mathrm{PH}$ was assessed graphically with weighted Schoenfeld residuals, which helped to inform of possible non-PH intervals that warranted analytical testing of a time-varying effect. To identify risk factors of mortality in multivariable analysis, redundancy in candidate predictors was assessed and any highly correlated variables were reduced appropriately. The remaining set of candidate predictor variables were screened for importance with stepwise variable selection using backward elimination and a liberal alpha level of 0.50 . 
Given the possibility that baseline factors played a role in the decision to treat with suture versus ring repair, this potential confounding on the association of repair technique with survival time (and other outcomes) was addressed using propensity-score matching. Multiple patient descriptors believed to be relevant to this selection were identified (eg, age, gender, body surface area, hypertension, diabetes, COPD, renal failure, NYHA class $\geq$ III, preoperative atrial fibrillation, preoperative transvenous pacemaker, ejection fraction, left atrial volume, left ventricular systolic and diastolic volume, RVSP, preoperative right ventricular dysfunction and size, preoperative MR grade $\geq 3$, preoperative TR grade $\geq 3$, MR etiology, MV replacement, maze procedure, and year of surgery) and simultaneously tested for an influence on the choice of repair in a multivariable logistic regression model, with suture repair as the dependent variable. For this analysis, missing data were imputed to retain all patients in the fitted model. The estimated propensity score, based on the logit-transformed predicted probability of receiving a suture repair, was then used to identify suitable matches between the 2 treatment groups. In particular, for each of the 194 patients with suture repair, all 221 patients treated with ring repair were searched to find a match with the closest propensity score. The final subset of all successful matches was then used to determine differences in survival and operative outcomes between the 2 TV repair groups: ring annuloplasty and DeVega annuloplasty (suture repair). The adequacy of the propensity-score matching in balancing these 2 groups on potential confounders was demonstrated by the comparison of individual factors between ring- and suture-matched patients.

In the subgroup of patients with at least 1 postoperative echocardiogram after hospital dismissal, longitudinal analysis for overall trends and technique-associated changes in TR grade were performed with the use of a mixed effects proportional-odds regression. This analysis fits a single model to all available measurements of TR grade over 5-year follow-up, with model inputs that included repair group, time of echocardiogram, and a group $\times$ time interaction, along with preoperative TR grade and propensity score as adjusting covariates. Group difference in trend was determined by testing the group $\times$ time interaction for significance. For ease of describing these trends, the marginal probability of each ordinal TR response was estimated (approximate integration over the random effect distribution using numeric quadrature) and plotted by group across follow-up time. Low frequency responses were collapsed into single categories for TR grades 0 and 1 and grades 3 and 4 . The analysis was repeated for other preselected candidate predictors of postoperative TR, including etiology of MV disease, RVSP, preoperative atrial fibrillation, presence of transvenous pacemaker, and MV repair (vs replacement), as well as for modeling ordinal responses of postoperative NYHA class. All data analyses were performed with the SAS statistical package (version 9.4, SAS institute, Cary, NC), with statistical significance of results determined based on an alpha level of 0.05 .

\section{RESULTS}

During the study interval, a total of 1350 adult patients (aged $>18$ years) underwent TV repair, and among them 898 patients had concomitant MV surgery. After considering the exclusion criteria, 415 patients were included from the overall population (Figure 1). Flexible rings used were Duran Ancore (Medtronic, Minneapolis, Minn), Carbomedics Annuloflex (Sorin Group USA, Arvada, Colo), and Cosgrove-Edwards (Edwards Lifesciences, Corp. Irvine, Calif). The most commonly used flexible ring size was the $25 \mathrm{~mm}$ Duran Ancore.

\section{Overall Sample $(N=415)$}

As shown in Table 1, baseline patient characteristics were generally similar between the $2 \mathrm{TV}$ repair groups, except for greater prevalence of preoperative transvenous pacemaker $(P=.05)$, slightly lower values of ejection fraction $(P=.028)$, and later year of surgical procedure $(P<.001)$ in the ring annuloplasty group.

Long-term mortality. Vital status was obtained on the overall sample $(\mathrm{N}=415)$ at a median follow-up period of 7.4 years (interquartile range [IQR], 3.6-11.6 years). Median survival time $(95 \%$ confidence interval) for patients who received ring and suture annuloplasty was 7.6 years (IQR, 6.2-9.5 years) and 8.1 years (IQR, 6.7-11.6 years), respectively. There was no significant difference between groups (suture annuloplasty HR, 0.81; 95\% CI, 0.61-1.08; $P=.152)$ in the unadjusted risk of long-term mortality (Figure 2, A, and Table E1). Predictors of increased mortality identified from multivariable analyses included older age at operation (per 10-year increase: HR, 1.05 ; 95\% CI, 1.04-1.07), diabetes (HR, 1.61; 95\% CI, 1.11-2.32), and preoperative right ventricular dysfunction (HR, 2.50; 95\% CI, 1.13-5.52) (Table 2).

Follow-up NYHA functional class. Based on 195 subjects with determination of NYHA status at least once over 5year follow-up, the majority of patients were in NYHA class I or II according to the first $(70 \%)$ or last $(66 \%)$ follow-up assessment. This reflected a significant degree of improvement compared with preoperative functional status ( $23 \%$ in NYHA class I or II), and NYHA class remained relatively stable and with no significant change during follow-up $(P=.279)$. Because no overall trend in postoperative NYHA measurements was detected, baseline factors were assessed for an association with postoperative levels, rather than changes, of functional status. In these analyses there was no association of repair technique with postoperative functional status $(P=.758)$, whereas ischemic etiology of MV disease and MV replacement (vs repair) were associated with worse levels of NYHA class during follow-up $(P=.029$ and $P=.025$, respectively).

TR on follow-up. From 210 patients who had at least 1 follow-up TTE within 5 years postoperatively, a total of 605 echocardiograms with TR measurements were available (average of $2.9 \pm 1.4$ per person), and among these patients, the mean follow-up time to the last echocardiogram was $2.7 \pm 1.4$ years (Figure 3 ). The probability of having different degrees of late postoperative TR was evaluated as a function of follow-up time (Figure 4). The probability of remaining in TR grade 0 or 1 declined comparably over time for ring- and suture-annuloplasty patients. At 1 year, the estimated probability of a TR grade 3 or 4 was $<10 \%$ for patients in both groups ( $5 \%$ for ring annuloplasty patients and $9 \%$ for suture annuloplasty patients). In the mixed effects proportional-odds model, the overall test for a treatment group difference in the trend of TR levels over follow-up was not significant $(P=.807)$.

The probability of having different degrees of TR over follow-up was also evaluated according to etiology of MV 


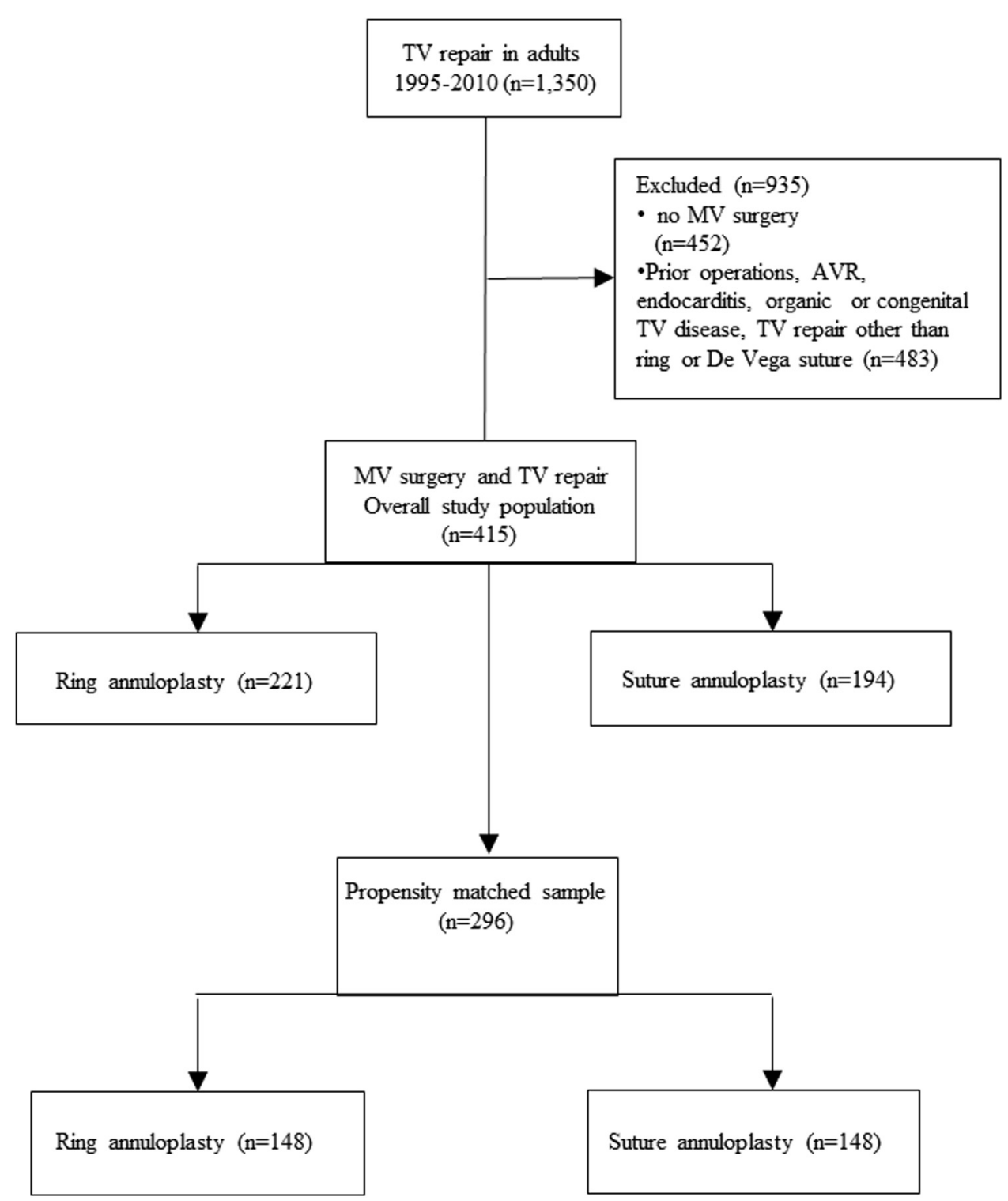

FIGURE 1. Diagram of patients entered into the study. $T V$, Triscuspid valve; $M V$, mitral valve; $A V R$, aortic valve replacement.

disease (Figure 5). Although patients with ischemic MR tended to show higher probabilities for TR $\geq 3$ during follow-up, the difference in trend between groups (degenerative vs rheumatic vs ischemic) for ordinal responses was not significant $(P=.857)$. Among other candidate predictors that were considered (ie, RVSP, preoperative or postoperative atrial fibrillation, pre- or postoperative transvenous pacemaker, or MV repair), none were shown to be significantly associated with postoperative TR trends.

TV reoperations. TV reoperations were performed in only 4 patients, and the initial TV repair was ring annuloplasty in 2 patients and De Vega suture method in 2 patients. In the 2 patients who underwent initial ring annuloplasty, 1 patient had TV replacement with a Hancock bioprosthesis
(Medtronic, Minneapolis, Minn) 3 years after the first procedure, and the other patient had TV replacement with a Carpentier-Edwards porcine valve (Edwards Lifesciences, Irvine, Calif) 2 years postoperatively. The cause of TV reoperation was severe functional TR related to failure of MV repair in 1 patient. The second patient had moderate pulmonary hypertension (systolic pulmonary artery pressure, $48 \mathrm{~mm} \mathrm{Hg}$ ) and developed atrial fibrillation after TV repair; at reoperation, he underwent TV replacement and a maze procedure. In the 2 patients with the previous De Vega suture TV repair, 1 had subsequent TV replacement with a Biocor prosthesis (St Jude Medical, St Paul, Minn) 1 year after the first operation, and the other had repeat TV annuloplasty with a Duran Ancore ring 2 years after initial suture 
TABLE 1. Patient preoperative and surgical characteristics by type of tricuspid valve repair in the unmatched population $(\mathrm{N}=415)$

\begin{tabular}{|c|c|c|c|}
\hline Variable & $\operatorname{Ring}(n=221)$ & Suture $(n=194)$ & $P$ value \\
\hline Age, $y$ & $72.0(65.0-79.0)$ & $71.0(62.0-77.0)$ & .160 \\
\hline Female gender & $138(62)$ & $121(62)$ & .988 \\
\hline Body surface area, $\mathrm{m}^{2}$ & $1.8(1.7-2.0)$ & $1.8(1.7-2.0)$ & .747 \\
\hline Hypertension & $143(65)$ & $121(62)$ & .622 \\
\hline Diabetes & $40(18)$ & $33(17)$ & .771 \\
\hline Chronic obstructive pulmonary disease & $40(18)$ & $46(24)$ & .159 \\
\hline Renal failure & $15(7)$ & $12(6)$ & .804 \\
\hline New York Heart Association functional class $\geq$ III & $177(80)$ & $148(76)$ & .348 \\
\hline Preoperative atrial fibrillation & $143(65)$ & $116(60)$ & .369 \\
\hline Preoperative pacemaker & $43(19)$ & $24(12)$ & .050 \\
\hline Ejection fraction, $\%$ & $58.5(50.0-65.0)$ & $60.0(52.0-65.0)$ & $.028 *$ \\
\hline Left atrium volume index $\mathrm{cc} / \mathrm{m}^{2}$ & $63.0(52.0-86.0)$ & $62.0(51.0-80.5)$ & .255 \\
\hline Left ventricular end systolic diameter, $\mathrm{mm}$ & $35.0(30.0-40.5)$ & $34.0(30.0-39.0)$ & .345 \\
\hline Left ventricular end diastolic diameter, mm & $52.0(47.0-57.0)$ & $52.0(47.0-57.0)$ & .911 \\
\hline Right ventricular systolic pressure, $\mathrm{mm} \mathrm{Hg}$ & $55.0(46.0-65.4)$ & $54.0(43.0-66.0)$ & .585 \\
\hline Preoperative right ventricular dysfunction $\geq 3$ & $9(5)$ & $4(3)$ & .325 \\
\hline Preoperative right ventricle size $\geq 3$ & $15(7)$ & $10(6)$ & .584 \\
\hline Preoperative mitral regurgitation $\geq 3$ & $174(79)$ & $143(74)$ & .229 \\
\hline Preoperative tricuspid regurgitation $\geq 3$ & $147(67)$ & $121(62)$ & .378 \\
\hline Etiology & & & .728 \\
\hline Degenerative & $122(55)$ & $103(53)$ & \\
\hline Ischemic & $35(16)$ & $28(14)$ & \\
\hline Rheumatic & $64(29)$ & $63(32)$ & \\
\hline Mitral valve replacement & $106(48)$ & $95(49)$ & .838 \\
\hline Maze & $55(25)$ & $47(24)$ & .876 \\
\hline Coronary artery bypass grafting & $46(21)$ & $49(25)$ & .282 \\
\hline Year of surgery & 2006 (2003-2009) & 2005 (2001-2008) & $<.001 *$ \\
\hline
\end{tabular}

annuloplasty. The causes of TV reoperation were TV annular dilation related to right heart failure in a patient receiving chronic hemodialysis, and severe functional TR due to failure of MV repair in the second patient (Table E2).

Regarding the pathology of MV disease in patients who underwent reoperation for recurrent TR, 1 patient with initial De Vega suture repair had rheumatic MV disease, and all other patients had degenerative MV disease.

\section{Propensity-matched Groups}

To account for baseline differences between the 2 treatment groups, propensity matching was performed to identify 148 matched pairs. There were no important differences between these matched groups with regard to baseline characteristics, demonstrating the efficacy of the propensity matching (Table 3). Among these patients, the median age was 71 years in each group, and the majority

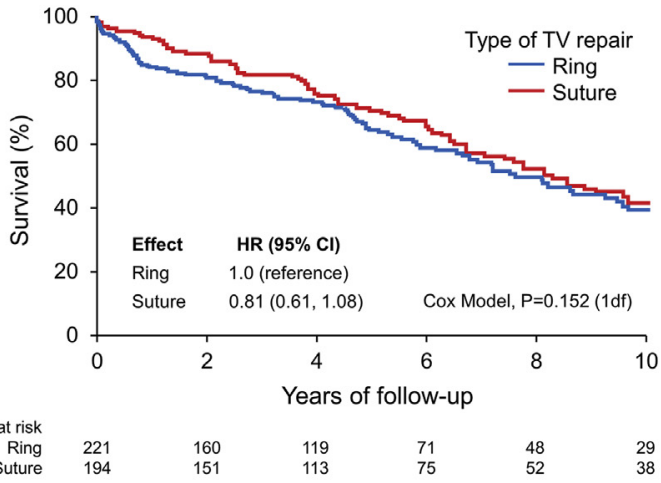

A

\begin{tabular}{rllllll} 
Ring & 221 & 160 & 119 & 71 & 48 & 29 \\
Suture & 194 & 151 & 113 & 75 & 52 & 38 \\
\hline & & & & & &
\end{tabular}

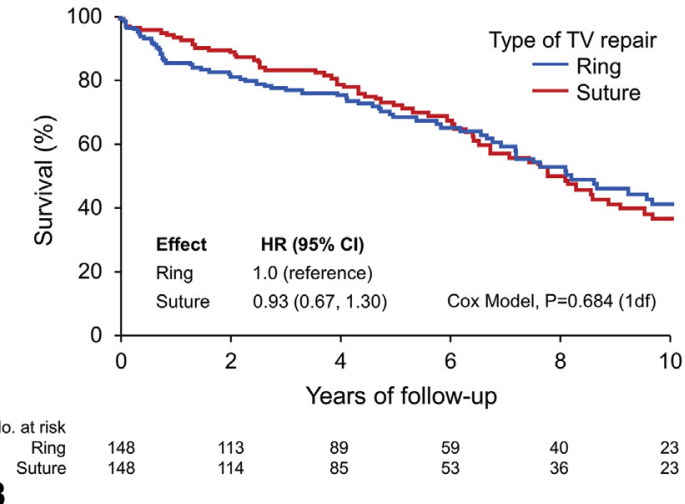

FIGURE 2. Panel A, Overall survival following tricuspid valve (TV) repair with mitral valve surgery. There was no significant difference in overall survival of patients having flexible ring (blue line) versus De Vega suture (red line) TV annuloplasty. Panel B, Survival of patients in the propensity-matched cohorts. Again, outcomes were not different for the 2 annuloplasty groups. HR, Hazard ratio; CI, confidence interval. 
TABLE 2. Predictors of long-term mortality for the overall sample $(\mathrm{N}=\mathbf{4 1 5})$

\begin{tabular}{lcr}
\hline \multicolumn{1}{c}{ Variable } & Multivariable result & $\boldsymbol{P}$ value \\
\hline Age (per 10-y increase) & $1.05(1.04-1.07)$ & $<. \mathbf{0 0 1}$ \\
Body surface area & $1.65(0.89-3.05)$ & .111 \\
Hypertension & $1.08(0.78-1.51)$ & .644 \\
Diabetes & $1.61(1.11-2.32)$ & $\mathbf{. 0 1 1}$ \\
Chronic obstructive pulmonary disease & $1.39(0.97-1.99)$ & .074 \\
Renal failure & $1.28(0.79-2.09)$ & .316 \\
Ejection fraction (per 10\% increase) & $0.92(0.81-1.05)$ & .228 \\
Preoperative right ventricular & $2.50(1.13-5.52)$ & $\mathbf{. 0 2 4}$ \\
$\quad$ dysfunction $\geq 3$ vs $<3 *$ & & \\
Preoperative right ventricle size $\geq 3$ & $1.93(0.99-3.75)$ & .137 \\
$\quad$ vs $<3 *$ & & \\
Etiology & & \\
$\quad$ Degenerative & $1.0($ referent $)$ & \\
$\quad$ Ischemic & $1.36(0.89-2.09)$ & \\
$\quad$ Rheumatic & $1.19(0.82-1.74)$ & \\
\hline
\end{tabular}

Values are presented as hazard ratio $(95 \%$ confidence interval). *An additional category to indicate missing data was included to assess the overall association with all subjects retained. Although the missing level was included among all pairwise contrasts analyzed, only estimates for the contrast of observed levels (ie, $\geq 3$ vs $<3$ ) are reported. Bold indicates $P$ values that achieves statistical significance.

had preserved ejection fractions. The main etiology for MV regurgitation was degenerative disease, and the MV was repaired in almost half of patients in each group.

Operative mortality and postoperative outcome variables did not differ between propensity-matched groups, with the exceptions of increased aortic crossclamp time (median, 67.5 vs 48.0 minutes; $P<.001)$ and cardiopulmonary bypass time (median, 105.5 vs 82.0 minutes; $P<.001$ ) for the ring annuloplasty group (Table 4).

Median survival time $(95 \% \mathrm{CI})$ for matched ring and suture annuloplasty groups was 8.1 years (range, 6.910.2 years) and 7.8 years (range, $6.5-9.1 ; P=.684$ years), respectively. Survival curves of propensity-matched patients having suture or ring annuloplasty are shown in Figure 2, $B$, and Table E1. For testing the association of annuloplasty method with survival time among matched pairs

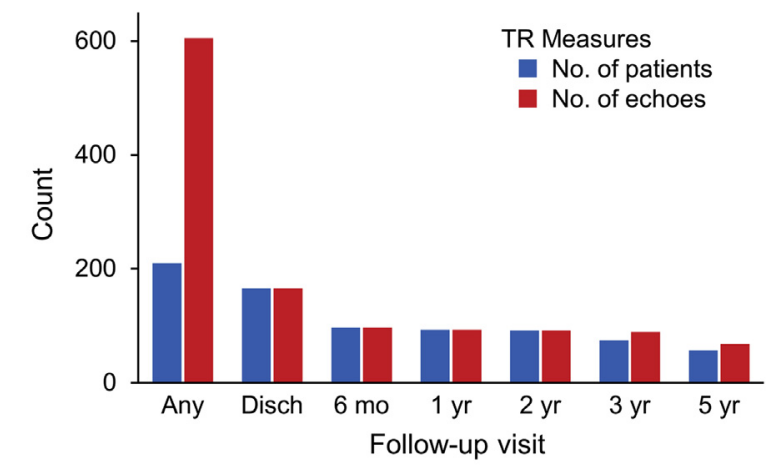

$\begin{array}{llllllll}\text { No. of patients } & 210 & 166 & 97 & 93 & 92 & 75 & 57 \\ \text { No. of echoes } & 605 & 166 & 97 & 93 & 92 & 89 & 68\end{array}$

FIGURE 3. Numbers of echocardiograms available for assessment of tricuspid regurgitation (TR) during follow-up.

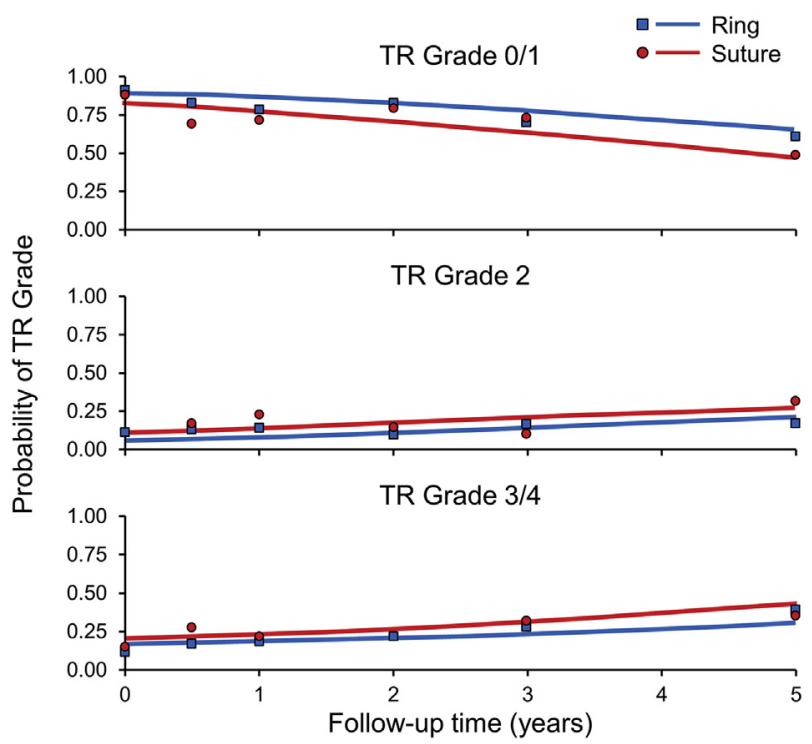

FIGURE 4. Probability of being in different tricuspid regurgitation $(T R)$ grades after ring or De Vega suture annuloplasty. Upper panel, The probability for remaining in grade 0 or 1 over the follow-up time. Middle panel, The probability of being in grade 2 . Lower panel, The probability of being in grade 3 or 4 . In the mixed effects proportional odds model, the overall test for a group difference in the trend of TR levels over follow-up was not significant $(P=.807)$.

in Cox regression, the model assumption of $\mathrm{PH}$ was assessed for validity using both graphic and analytical methods. Despite some evidence that the ratio of hazards between ring- and suture-treated groups differed across early to late follow-up $(P=.033$ comparing the HR estimated in first year vs after 1 year [Table E3]), testing for $\mathrm{PH}$ was sensitive to the choice of follow-up intervals and was insignificant when assessed as a continuous scale of time. For more details on the tests and results pertaining to non-PH patterns, including interval-specific estimates for treatment effect on mortality, see Supplemental Data. Thus, we report the overall effect for repair technique as an average HR throughout all event times, which indicated annuloplasty method was not associated with risk of longterm mortality (suture annuloplasty HR, $0.93 ; 95 \% \mathrm{CI}$, $0.67-1.30 ; P=.68$ ) among propensity-matched pairs (Table 5).

\section{DISCUSSION}

TV regurgitation is a common echocardiographic finding that is present in $80 \%$ to $90 \%$ of normal individuals. ${ }^{12}$ Most often, the volume of TR is small and has little influence on cardiac function. In many patients with significant TR, TV disease is secondary to other cardiac conditions. Functional TR usually results from left-sided heart disease, most often MV stenosis or regurgitation. Functional TV leakage is caused by tricuspid annular dilatation, along with increased preload, afterload, and right ventricular dysfunction. It is 


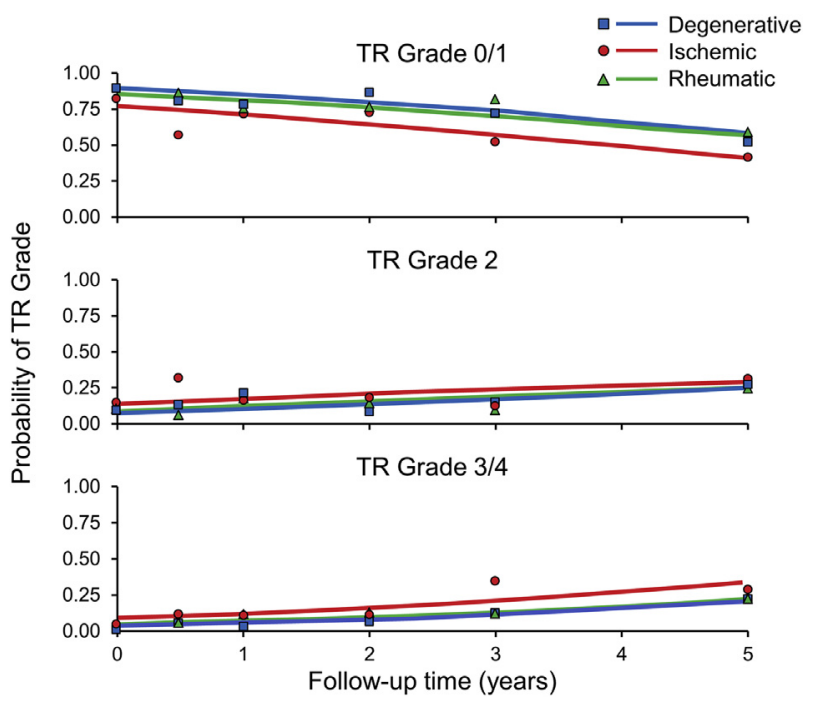

FIGURE 5. Probability of being in different tricuspid regurgitation (TR) grade after tricuspid valve repair (either ring or suture annuloplasty) stratified by etiology of mitral regurgitation. Upper panel describes the probability for remaining grade 0 or 1 . The middle panel illustrates the probability of being grade 2 , and lower panel illustrates the probability of being grade 3 or 4 .

clear that other hemodynamic problems often accompany TR and may complicate the assessment of the influence of TR on the patient.

There is considerable controversy among surgeons regarding the optimal treatment of functional TV regurgitation. Two recent clinical studies suggest that late results of TV repair with ring annuloplasty are superior to repair with suture annuloplasty. In the study reported by McCarthy and colleagues, ${ }^{1}$ the surgical outcomes of TV annuloplasty using 4 techniques were compared in patients undergoing operation between 1990 and 1999. Severity of residual regurgitation was similar with the 4 techniques early, but over time residual TR rose more rapidly in patients with De Vega $(P=.002)$ repair and Peri-Guard (Synovis Surgical Innovations, A Division of Synovis Life Technologies, Inc. St Paul, Minn $)(P=.0009)$ band annuloplasty compared with ring methods. In another report by Guenther and colleagues, ${ }^{13} 10$-year survival after TV repair with either a ring annuloplasty or De Vega annuloplasty were $46 \% \pm 7 \%$, and $39 \% \pm 3 \%(P=.01)$, and freedom from TV reoperation after TV repair with De Vega suture annuloplasty was $88 \%$ compared with $98 \%$ after a ring annuloplasty $(P=.034)$. The authors concluded that a ring annuloplasty was associated with improved survival and lower rate of reoperation than a suture annuloplasty.

Our experience in patients undergoing TV repair at the time of MV surgery differs from these previous reports because we found no difference in patient survival comparing groups with De Vega suture and ring TV annuloplasty. Results of patient survival were similar for the overall cohort as well as propensity-matched patients. Further, there was no difference in patient outcome with suture or ring annuloplasty in regard to late functional status, progression of late TR, or TV reoperation.

There are several possible explanations for discordance in our experience versus prior studies. Era of operation may have influenced the results. In both of the previously mentioned investigations, ${ }^{1,13}$ ring annuloplasties were used in the later periods of the study. Further, the methods used for suture annuloplasty and the degree of annular reduction was highly variable. For example, Guenther and colleagues $^{13}$ described use of a 3-0 polypropylene suture from anteroseptal commissure to posteroseptal commissure to restrict the orifice to 3 finger breadths. As discussed below, we favor a much tighter initial reduction in TV orifice with pledgeted 0-Prolene suture (Ethicon, Somerville, $\mathrm{NJ}$ ) that encompasses the annulus from the anteroseptal commissure to a point on the septal leaflet opposite the midportion of the coronary sinus. Also, both series included patients with previous cardiac surgery $(51 \%$ in the study by McCarthy and colleagues, ${ }^{1} 34 \%$ in the study by Guenther and colleagues ${ }^{13}$ ) and concomitant aortic surgery. The study by Guenther and colleagues ${ }^{13}$ included a heterogenous group of patients with TR, including patients with TV endocarditis and patients with TR due to trauma.

Other investigations have shown no important differences in clinical outcomes of suture annuloplasty versus ring annuloplasty. ${ }^{14,15}$ Moreover, in a study of 299 patients with multivalvular rheumatic heart disease, Sarralde and colleagues ${ }^{16}$ reported better late survival of patients undergoing De Vega repair compared with those having ring annuloplasty for TV repair. All of the aforementioned studies should be interpreted cautiously because of wide variation in surgeons' attitudes toward patient selection and choice of procedure. TV repair with ring annuloplasty is popular now, and selection of this technique may be influenced by surgeons' experience with use of rings for MV repair.

In the present study there was no difference in early mortality of patients receiving suture annuloplasty versus ring annuloplasty, and this contrasts with the findings of a meta-analysis by Parolari and colleagues. ${ }^{5}$ In that report, patients who underwent ring annuloplasty had decreased operative mortality (30-day or in-hospital mortality). Their conclusions are driven mainly from observational studies. ${ }^{13,15,17}$ In a subgroup meta-analysis with only randomized studies, there was no difference in early mortality (relative risk, $0.91 ; P=.8$ ).

We focused on the surgical outcomes of TV repair in patients with functional TV regurgitation undergoing MV surgery. In our practice and in most centers in North America, degenerative etiologies causing regurgitation are the most common findings in patients undergoing MV surgery. ${ }^{18}$ We excluded patients who underwent prior cardiac surgery and those who had concomitant aortic valve repair or 
TABLE 3. Patient preoperative and surgical characteristics by type of tricuspid valve (TV) repair in the propensity-matched sample (N $=296$ )

\begin{tabular}{|c|c|c|c|}
\hline Variable & $\operatorname{Ring}(n=148)$ & Suture $(n=148)$ & $P$ value \\
\hline Age, $y$ & $71.0(63.5-78.0)$ & $71.0(64.0-78.0)$ & .822 \\
\hline Female gender & $97(66)$ & $91(61)$ & .469 \\
\hline Body surface area, $\mathrm{m}^{2}$ & $1.8(1.7-2.0)$ & $1.8(1.7-2.0)$ & .749 \\
\hline Hypertension & $96(65)$ & $89(60)$ & .401 \\
\hline Diabetes & $25(17)$ & $23(16)$ & .752 \\
\hline Chronic obstructive pulmonary disease & $34(23)$ & $30(20)$ & .572 \\
\hline Renal failure & $10(7)$ & $9(6)$ & .813 \\
\hline New York Heart Association functional class $\geq$ III & $115(78)$ & $109(74)$ & .416 \\
\hline Preoperative atrial fibrillation & $87(59)$ & $87(59)$ & .944 \\
\hline Pacemaker & $21(14)$ & $16(11)$ & .380 \\
\hline Ejection fraction, $\%$ & $60.0(55.0-65.0)$ & $60.0(50.5-66.0)$ & .945 \\
\hline Left atrium volume index, $\mathrm{cc} / \mathrm{m}^{2}$ & $62.0(52.0-84.0)$ & $64.0(52.0-81.0)$ & .850 \\
\hline Left ventricular end systolic diameter, $\mathrm{mm}$ & $34.0(30.0-39.0)$ & $34.0(30.0-39.0)$ & .888 \\
\hline Left ventricular end diastolic diameter, $\mathrm{mm}$ & $52.0(47.0-58.0)$ & $52.0(47.0-57.0)$ & .579 \\
\hline Right ventricular systolic pressure, $\mathrm{mm} \mathrm{Hg}$ & $56.0(46.0-67.0)$ & $52.0(43.0-66.0)$ & .157 \\
\hline Preoperative right ventricular dysfunction $\geq 3$ & $6(5)$ & $3(2)$ & .326 \\
\hline Preoperative right ventricle size $\geq 3$ & $9(6)$ & $9(7)$ & .962 \\
\hline Preoperative mitral regurgitation $\geq 3$ & $115(78)$ & $114(77)$ & .890 \\
\hline Preoperative tricuspid regurgitation $\geq 3$ & $93(63)$ & $95(64)$ & .809 \\
\hline Etiology & & & .970 \\
\hline Degenerative & $81(55)$ & $83(56)$ & \\
\hline Ischemic & $19(13)$ & $18(12)$ & \\
\hline Rheumatic & $48(32)$ & $47(32)$ & \\
\hline Mitral valve replacement & $74(50)$ & $73(49)$ & .907 \\
\hline Maze & $33(22)$ & $41(28)$ & .283 \\
\hline Coronary artery bypass grafting & $31(21)$ & $30(20)$ & .886 \\
\hline Year of surgery & $2005(2003-2008)$ & $2005(2003-2008)$ & .838 \\
\hline
\end{tabular}

Values are presented as $\mathrm{n}(\%)$, or median (interquartile range).

replacement. MV reoperations may be difficult technically due to poor exposure and may require additional crossclamp time for explantation of a prosthesis. These factors may influence the surgeon to choose suture rather than ring annuloplasty to minimize times of aortic crossclamping and cardiopulmonary bypass. Thus, we excluded these patients to eliminate this potential bias.
Unlike most other studies, suture annuloplasty was used throughout the study period, thus minimizing the potential bias of era of operation. Regarding the technique of De Vega suture annuloplasty, we prefer initial "overcorrection" at the time that the suture is tied. The ideal degree of narrowing is somewhat subjective, but we routinely narrow the orifice to a diameter of $2 \mathrm{~cm}$. We have not

TABLE 4. Operative and postoperative outcomes for the propensity-matched sample $(\mathbf{N}=296)$

\begin{tabular}{|c|c|c|c|}
\hline Variable & Ring $(n=148)$ & Suture $(n=148)$ & $P$ value \\
\hline Operative mortality & $3(2)$ & $2(1)$ & .652 \\
\hline Cardiopulmonary bypass time, min & $105.5(77.0-133.0)$ & $82.0(64.0-112.0)$ & $<.001 *$ \\
\hline Aortic crossclamp time, min & $67.5(49.0-88.0)$ & $48.0(36.0-70.0)$ & $<.001 *$ \\
\hline Total time in intensive care unit, $\mathrm{h} \dagger$ & $45.0(24.5-92.0)$ & $42.5(23.5-70.0)$ & .461 \\
\hline Prolonged ventilation & $29(20)$ & $27(18)$ & .767 \\
\hline Atrial fibrillation at discharge & $72(51)$ & $67(48)$ & .592 \\
\hline Tricuspid regurgitation $\geq 3$ at discharge & $3(2)$ & $2(1)$ & .663 \\
\hline Mitral regurgitation $\geq 3$ at discharge & $1(1)$ & $0(0)$ & 1.000 \\
\hline Ejection fraction at discharge, $\%$ & $56.5(46.0-61.0)$ & $55.0(49.0-63.0)$ & .584 \\
\hline Right ventricular systolic pressure at discharge, $\mathrm{mm} \mathrm{Hg} \dagger$ & $43.6(36.0-54.0)$ & $44.0(34.0-51.0)$ & 676 \\
\hline Right ventricular dysfunction $\geq 3$ at discharge $\ddagger$ & $11(9)$ & $11(9)$ & .953 \\
\hline Right ventricle size $\geq 3$ at discharge $\neq$ & $3(2)$ & $3(2)$ & .978 \\
\hline Left ventricular diastolic diameter at discharge, $\mathrm{mm}^{\dagger}$ & $50.5(46.0-56.0)$ & $50.0(46.0-55.0)$ & .281 \\
\hline
\end{tabular}

Outcome variables are missing in $<5 \%$ of subjects, unless indicated otherwise. Values are presented as n ( $\%$ ) or median (interquartile range). $* P<.05$. $\dagger$ Missing data in $16 \%$ to $25 \%$. ‡Missing data in $5 \%$ to $15 \%$. 
TABLE 5. Unmatched and propensity-adjusted effect of treatment repair type on overall mortality

\begin{tabular}{lcccc}
\hline Treatment effect & $\begin{array}{c}\text { All patients } \\
(\mathbf{N}=\mathbf{4 1 5})\end{array}$ & $\begin{array}{c}\boldsymbol{P} \\
\text { value }\end{array}$ & $\begin{array}{c}\text { Propensity-score } \\
\text { matched }(\mathbf{n}=\mathbf{2 9 6})\end{array}$ & $\begin{array}{c}\boldsymbol{P} \\
\text { value }\end{array}$ \\
\hline Suture (vs ring) & $0.81(0.61-1.08)$ & .152 & $0.93(0.67-1.3)$ & .684 \\
\hline
\end{tabular}

Values are presented as hazard ratio $(95 \%$ confidence interval). $*$ Without timeinterval interaction.

consistently used an obturator ${ }^{19}$ or valve sizer to aid in securing the annuloplasty suture, but if a device is used, we prefer an 18- or 20-Hegar dilator. Too often, surgeons are concerned about narrowing the TV orifice when, in fact, the suture annuloplasty loosens somewhat after cardiac rhythm and blood flow across the valve are restored. For example, securing the annuloplasty suture around a 20Hegar dilator would leave a TV orifice of $3.1 \mathrm{~cm}^{2}$, well above the generally accepted $1-\mathrm{cm}$ area associated with significant stenosis. ${ }^{20}$ In our experience, it is rare for the transvalvular gradient after tricuspid annuloplasty to be greater than 2 to $3 \mathrm{~mm} \mathrm{Hg}$, and, most often, there is no gradient. Further, if mild TV stenosis does persist, this is usually well tolerated ${ }^{21}$ and may decrease with time.

Clearly, both suture annuloplasty and ring annuloplasty yield good results, but suture annuloplasty has 2 clear advantages. First, it can be performed simply and rapidly; this may be important in some patients. In the present study, times of cardiopulmonary bypass and aortic crossclamping were 20 minutes less in the De Vega annuloplasty group compared with patients undergoing ring annuloplasty. One study reported a higher incidence of postoperative low cardiac output with ring annuloplasty compared with suture repair, possibly related to longer aortic crossclamp time and bypass time. ${ }^{15}$ An additional advantage of the suture annuloplasty is low cost compared with commercially prepared prosthetic devices. ${ }^{22}$

In the present study of patients with repaired functional TV regurgitation, we found no difference in the late development of recurrent TR according to MV pathology (rheumatic vs degenerative vs ischemic). These data should be interpreted cautiously because we had echocardiographic follow-up only in 210 of 415 eligible patients. Important too will be future analyses that correlate residual TR with recurrent MV dysfunction according to the type of mitral pathology.

Functional TV regurgitation may be associated with pulmonary hypertension, RV dysfunction, and atrial fibrillation. Left atrial and pulmonary venous hypertension due to MV stenosis and/or regurgitation often causes pulmonary hypertension. Long-standing pulmonary hypertension may lead to right ventricular dysfunction and remodeling, which, in turn, produces tricuspid annular dilation, papillary muscle displacement, tethering of the TV leaflet, and TV leakage. ${ }^{23}$ TR itself further increases RV size and may cause right ventricular dysfunction, more TV annular dilatation, leaflet tethering, and worsening TR. Increased left atrial size and pressure might also cause atrial fibrillation, which in turn causes right atrial dilation leading to further tricuspid annular dilation. Reports suggest that patients who have concomitant successful maze procedure during their MV surgery have significantly less TR at late follow-up. ${ }^{24,25}$ In the present series there was no association between the above 3 factors and postoperative follow-up TR $\geq 3$ change. But our analysis only included preoperative information on atrial fibrillation, RV dysfunction, and RVSP, and it is unknown whether these factors developed late after TV repair and contributed to recurrent TR. Inclusion of preoperative TV anatomic information, such as mode of leaflet coaptation, may further improve our understanding and predictors for ring or suture annuloplasty failure. ${ }^{26}$

Long-term mortality of patients who underwent suture and ring annuloplasty was similar in the overall and PM cohorts. In the multivariable analysis, older age, diabetes, and preoperative RV dysfunction were independent predictors of late mortality.

In this series, reoperation for recurrent TR was performed infrequently (4 patients), and there was no difference in the incidence of reoperation according to the type of TV repair. Among the patients who did undergo reoperation, 3 had bioprosthesis implanted with satisfactory results. Late complications of tissue degeneration of bioprostheses may be reduced in the tricuspid position where closing pressure is lower than that of mitral or aortic valves. ${ }^{27}$ As has been stated previously, that late reoperation rates may underestimate late failure of valve repair because of reluctance to refer patients due to the anticipated high risk of reoperation. ${ }^{1}$

\section{Study Limitations}

This study is retrospective with the usual limitations of such investigations. There were no consistently measured echocardiographic data available to quantify TV tethering height or tricuspid annular size, for example. Hemodynamic parameters on follow-up TTE were not measured regularly, and therefore we could not assess the effect of postoperative pulmonary hypertension on the recurrence of TR. The average time to last echocardiographic assessment of TR was relatively short, and it is possible that longer followup would reveal differences in outcome. The type of TV repair was determined by surgeon's preference. We were unable to correlate recurrence of TR to residual MR and to late functional status. Although we used flexible rings with demonstrated beneficial long-term results, newergeneration rings were not evaluated in this study and therefore our results may not be representative for everyday practice in institutions that use those devices.

\section{CONCLUSIONS}

In this experience with patients undergoing repair of functional TR during MV surgery, late patient survival 
and durability of TV repair are similar among patients undergoing ring annuloplasty and De Vega suture annuloplasty. The etiology of MV disease did not influence residual or recurrent TV regurgitation.

\section{Conflict of Interest Statement}

Authors have nothing to disclose with regard to commercial support.

\section{References}

1. McCarthy PM, Bhudia SK, Rajeswaran J, Hoercher KJ, Lytle BW, Cosgrove DM, et al. Tricuspid valve repair: durability and risk factors for failure. J Thorac Cardiovasc Surg. 2004;127:674-85.

2. Lewis MJ, Ginns JN, Ye S, Chai P, Quaegebeur JM, Bacha E, et al. Postoperative tricuspid regurgitation after adult congenital heart surgery is associated with adverse clinical outcomes. J Thorac Cardiovasc Surg. 2016;151:460-5.

3. David TE, David CM, Manhiolt C. When is tricuspid valve annuloplasty necessary during mitral valve surgery? J Thorac Cardiovasc Surg. 2015;150:1043-4.

4. Khorsandi M, Banerjee A, Singh H, Srivastava AR. Is a tricuspid annuloplasty ring significantly better than a De Vega's annuloplasty stitch when repairing severe tricuspid regurgitation? Interact Cardiovasc Thorac Surg. 2012;15:129-35.

5. Parolari A, Barili F, Pilozzi A, Pacini D. Ring or suture annuloplasty for tricuspid regurgitation? A meta-analysis review. Ann Thorac Surg. 2014;98:2255-63.

6. De Vega NG. [Selective, adjustable and permanent annuloplasty. An original technic for the treatment of tricuspid insufficiency]. Rev Esp Cardiol. 1972;25: 555-6.

7. Rabago G, De Vega NG, Castillon L, Moreno T, Fraile J, Azpitarte J, et al. The new De Vega technique in tricuspid annuloplasty (results in 150 patients). J Cardiovasc Surg. 1980;21:231-8.

8. Owan TE, Hodge DO, Herges RM, Jacobsen SJ, Roger VL, Redfield MM. Trends in prevalence and outcome of heart failure with preserved ejection fraction. N Engl J Med. 2006;355:251-9.

9. Edmunds LH Jr, Clark RE, Cohn LH, Grunkemeier GL, Miller DC, Weisel RD. Guidelines for reporting morbidity and mortality after cardiac valvular operations. Ad Hoc Liaison Committee for Standardizing Definitions of Prosthetic Heart Valve Morbidity of The American Association for Thoracic Surgery and The Society of Thoracic Surgeons. J Thorac Cardiovasc Surg. 1996;112:708-11.

10. Zoghbi WA, Enriquez-Sarano M, Foster E, Grayburn PA, Kraft CD, Levine RA, et al. Recommendations for evaluation of the severity of native valvular regurgitation with two-dimensional and Doppler echocardiography. J Am Soc Echocardiogr. 2003;16:777-802

11. Lang RM, Bierig M, Devereux RB, Flachskampf FA, Foster E, Pellikka PA, et al. Recommendations for chamber quantification: a report from the American Society of Echocardiography's Guidelines and Standards Committee and the Chamber Quantification Writing Group, developed in conjunction with the European Association of Echocardiography, a branch of the European Society of Cardiology. J Am Soc Echocardiogr. 2005;18:1440-63.
12. Singh JP, Evans JC, Levy D, Larson MG, Freed LA, Fuller DL, et al. Prevalence and clinical determinants of mitral, tricuspid, and aortic regurgitation (the Framingham Heart Study). Am J Cardiol. 1999;83:897-902.

13. Guenther T, Mazzitelli D, Noebauer C, Hettich I, Tassani-Prell P, Voss B, et al Tricuspid valve repair: is ring annuloplasty superior? Eur J Cardiothorac Surg. 2013;43:58-65; discussion 65.

14. Giamberti A, Chessa M, Ballotta A, Varrica A, Agnetti A, Frigiola A, et al. Functional tricuspid valve regurgitation in adults with congenital heart disease: an emerging problem. J Heart Valve Dis. 2011;20:565-70.

15. Carrier M, Pellerin M, Guertin M-C, Bouchard D, Hébert Y, Perrault LP, et al Twenty-five years' clinical experience with repair of tricuspid insufficiency. J Heart Valve Dis. 2004;13:952-6.

16. Sarralde JA, Bernal JM, Llorca J, Pontón A, Diez-Solorzano L, GiménezRico JR, et al. Repair of rheumatic tricuspid valve disease: predictors of very long-term mortality and reoperation. Ann Thorac Surg. 2010;90:503-8.

17. Navia JL, Nowicki ER, Blackstone EH, Brozzi NA, Nento DE, Atik FA, et al Surgical management of secondary tricuspid valve regurgitation: annulus, commissure, or leaflet procedure? J Thorac Cardiovasc Surg. 2010;139: 1473-82.e5.

18. Olson LJ, Subramanian R, Ackermann DM, Orszulak TA, Edwards WD. Surgica pathology of the mitral valve: a study of 712 cases spanning 21 years. Mayo Clin Proc. 1987;62:22-34.

19. Hwang HY, Chang HW, Jeong DS, Ahn H. De Vega annuloplasty for functional tricuspid regurgitation: concept of tricuspid valve orifice index to optimize tricuspid valve annular reduction. J Korean Med Sci. 2013;28:1756-61.

20. Baumgartner H, Hung J, Bermejo J, Chambers JB, Evangelista A, Griffin BP et al. Echocardiographic assessment of valve stenosis: EAE/ASE recommendations for clinical practice. J Am Soc Echocardiogr. 2009;22:1-23. quiz 101-2.

21. Antunes MJ, Barlow JB. Management of tricuspid valve regurgitation. Heart 2007;93:271-6.

22. Shinn SH, Schaff HV. Evidence-based surgical management of acquired tricuspid valve disease. Nature Rev Cardiol. 2013;10:190-203.

23. Fukuda S, Song J-M, Gillinov AM, McCarthy PM, Daimon M, Kongsaerepong V, et al. Tricuspid valve tethering predicts residual tricuspid regurgitation after tricuspid annuloplasty. Circulation. 2005;111:975-9.

24. Kim H-K, Kim Y-J, Kim K-I, Jo S-H, Kim K-B, Ahn H, et al. Impact of the maze operation combined with left-sided valve surgery on the change in tricuspid regurgitation over time. Circulation. 2005;112:I14-9.

25. Stulak JM, Schaff HV, Dearani JA, Orszulak TA, Daly RC, Sundt TM III. Restoration of sinus rhythm by the Maze procedure halts progression of tricuspid regurgitation after mitral surgery. Ann Thorac Surg. 2008;86:40-4; discussion 44-5.

26. Dreyfus GD, Martin RP, Chan KMJ, Dulguerov F, Alexandrescu C. Functional tricuspid regurgitation: a need to revise our understanding. J Am Coll Cardiol. 2015;65:2331-6.

27. Fleming WH, Sarafian LB, Moulton AL, Robinson LA, Kugler JD. Valve replacement in the right side of the heart in children: long-term follow-up. Ann Thorac Surg. 1989;48:404-8.

Key Words: tricuspid valve repair, tricuspid valve regurgitation 


\section{APPENDIX. ASSESSMENT OF PROPORTIONAL HAZARDS ASSUMPTION}

Visual inspection of the weighted Schoenfeld residuals plot suggested the possibility of a time-varying effect on the hazard function of mortality, as shown by a fairly constant decreased hazard ratio for the suture group over early follow-up (for approximately the first year after surgery) and a generally increasing hazard ratio thereafter (Figure E1). To further assess the importance of this treatment effect as a function of time, time-dependent variables were defined to characterize 2 to 3 nonoverlapping followup intervals, as guided by the plot, and construct time $\times$ group interactions in Cox regression. A formal test for proportional hazards was then derived by testing this interaction coefficient for significance, which is consistent with testing whether the within-interval estimates of the hazard ratio differ. When based on a single contrast of early versus late follow-up with the breakpoint set at 1 year, a significant time $\times$ group interaction was detected $(P=.033)$ which indicates the 2 groups did not satisfy proportional hazards. However, this approach appeared to be sensitive to the choice of intervals, because a 3-interval categorization that stratified the late period into before and after 5- year intervals resulted in a nonsignificant interaction $(P=.108)$. Use of a different 3-interval version of time, classified as $<1$ year, 1 to 10 years, and $>10$ years, did result in a significant interaction $(P=.037)$, although this timevarying effect of suture does not appear to follow a monotonic pattern (hazard ratio for early, intermediate, and late phases of $0.45,1.26$, and 0.53 ) and is thus difficult to interpret. Furthermore, based on a test for interaction using a continuous form of time, there is no evidence of an increasing or decreasing trend over time in the hazard ratio $(P=.334)$. These results suggest, at most, that the ratio of hazards within the first year differ from that after the first year, and thus we report interval-specific estimates for repair technique to summarize early and late effects on survival. The inference for these interval-specific results is that suture repair may be associated with improved survival in the first year since surgery compared with ring repair, and no significant difference in survival after 1-year followup. Given the lack of robust evidence or a clinical explanation supporting this non-proportional hazards pattern, a single overall effect for repair technique is reported in the primary results section based on the average hazard ratio throughout all event times. 


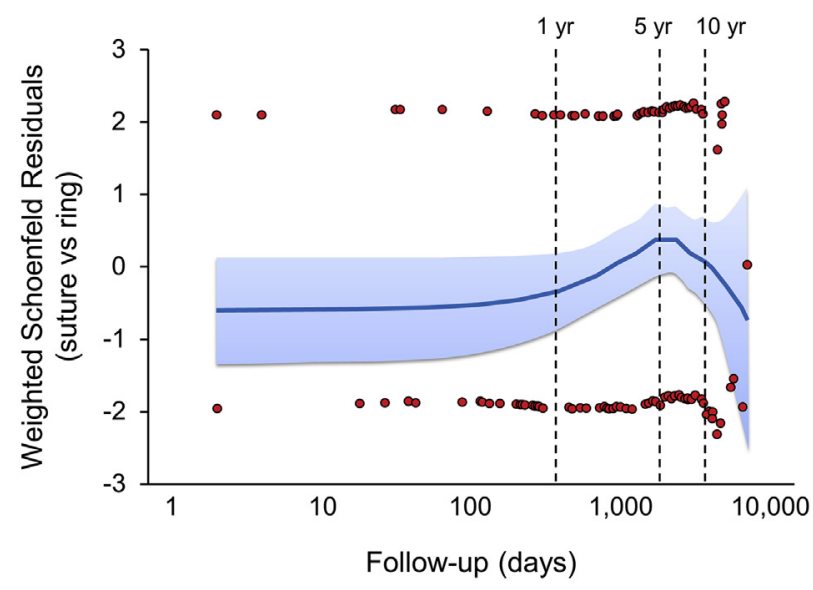

FIGURE E1. Graphical inspection of proportional hazards assumption for suture (vs ring) repair. Weighted Schoenfeld residuals from Cox model plotted against follow-up time (log-scale) to assess whether survival patterns for the 2 matched groups have proportional hazards. Smoothed trends in the residuals (and $95 \%$ confidence bands) are based on loess estimator.
TABLE E2. Patients who underwent reoperation due to tricuspid valve regurgitation in the overall sample

\begin{tabular}{lccc}
\hline Patient & $\begin{array}{c}\text { Suture } \\
\text { or ring } \\
\text { annuloplasty }\end{array}$ & Cause for failure & Treatment \\
\hline 1 & S & $\begin{array}{c}\text { Annular dilatation } \\
\text { and RV failure } \\
\text { MV repair failure }\end{array}$ & Biocor bioprosthesis* \\
2 & S & $\begin{array}{c}\text { Duran Ancore ring } \dagger \\
\text { MV repair failure }\end{array}$ & $\begin{array}{c}\text { Hancock II bioprosthesis } \dagger \\
\text { Carpentier-Edwards } \\
3\end{array}$ \\
4 & R & $\begin{array}{c}\text { Pulmonary HT } \\
\text { and AFib }\end{array}$ & \begin{tabular}{c} 
bioprosthesis $\ddagger$ \\
\hline
\end{tabular} \\
\hline
\end{tabular}

$S$, De Vega suture annuloplasty; $R V$, right ventricular; $M V$, mitral valve; $R$, flexible ring annuloplasty; HT, hypertension; AFib, atrial fibrillation. *St Jude, St Paul, Minn. $\dagger$ Medtronic, Minneapolis, Minn. $\ddagger$ Edwards Lifesciences, Irvine, Calif.
TABLE E1. Survival of overall and propensity-matched patients after tricuspid valve repair

\begin{tabular}{lcc}
\hline \multicolumn{1}{c}{ Type of repair } & Time, $\mathbf{y}$ & Survival \\
\hline Overall patients & & \\
$\quad$ Ring annuloplasty & 5 & $64.2(57.2-71.1)$ \\
& 10 & $38.8(28.8-47.7)$ \\
$\quad$ Suture annuloplasty & 5 & $70.2(63.1-77.3)$ \\
& 10 & $41.2(32.1-50.3)$ \\
Propensity-matched patients & & \\
$\quad$ Ring annuloplasty & 5 & $67.9(59.9-75.9)$ \\
$\quad 10$ & $40.4(29.9-50.9)$ \\
$\quad$ Suture annuloplasty & 5 & $31.9(63.7-80.0)$ \\
& 10 & $35.9(25.1-46.6)$ \\
\hline
\end{tabular}

Survival values are presented as $\%$ (95\% confidence interval). 
TABLE E3. Propensity-adjusted effect of treatment repair type on long-term mortality

\begin{tabular}{ccccc}
\hline Effect & Interval & Unadjusted result $(\mathbf{N}=\mathbf{4 1 5})$ & $\begin{array}{c}\text { Propensity score-matched } \\
\text { result }(\mathbf{n}=\mathbf{2 9 8})\end{array}$ & $\begin{array}{c}\text { Propensity score-matched, } \\
\text { further adjusted result* } \\
\text { (n= 298) }\end{array}$ \\
\hline Suture (vs ring) & & Interaction, $P=.033$ & Interaction, $P=.031$ & $\begin{array}{c}\text { Interaction, } P=.051 \\
\end{array}$ \\
& $0-365$ & $0.44(0.23-0.84)$ & $0.45(0.20-0.98)$ & $0.43(0.18-1.02)$ \\
& $>365$ & $0.96(0.70-1.33)$ & $1.13(0.79-1.63)$ & $1.10(0.76-1.59)$ \\
\hline
\end{tabular}

*Effect of suture was further adjusted for potential risk factors for mortality; each factor included in the previous multivariable time-to-death model was included as an adjusting covariate (ie, age, body surface area, hypertension, diabetes, ejection fraction, preoperative right ventricle dysfunction and size, and etiology). 